\title{
Development of high power $X$-band semiconductor microwave switch for pulse compression systems of future linear colliders
}

\author{
Fumihiko Tamura and Sami G. Tantawi \\ Stanford Linear Accelerator Center, P.O. Box 4349, Stanford, California 94309
}

(Received 17 January 2002; published 28 June 2002)

\begin{abstract}
We describe concepts for high power semiconductor rf switches, designed to handle signals at $X$-band with power level near $100 \mathrm{MW}$. We describe an abstract design methodology and derive a general scaling law for these switches. We also present a design and experimental work of a switch operating at the $\mathrm{TE}_{01}$ mode in overmoded circular waveguides. The switch is composed of an array of tee junction elements that have a $p-i-n$ diode array window in the third arm.
\end{abstract}

DOI: 10.1103/PhysRevSTAB.5.062001

PACS numbers: $84.40 .-\mathrm{x}$

\section{INTRODUCTION}

In recent years there have been many suggestions for the rf pulse compression and power distribution systems for the Next Linear Collider [1]. One of the most attractive systems for high power pulse compression is the so-called SLED II system [2]. This system is based on storing the rf energy in resonant delay lines and switching, or discharging, it by means of phase manipulations. This system suffers from low efficiency at high compression ratios; the compression ratio being the ratio between the input pulse width and the output pulse width. Theoretical studies have shown that the efficiency of these systems can be improved if one discharges the system be means of an active switch [3].

The development of passive rf pulse compression systems has led to the invention of many novel rf components and systems which must handle up to $600 \mathrm{MW}$ of pulsed power at $X$-band. These include passive waveguide components [4] and nonreciprocal devices [5]. The high power handling capabilities of these devices have been achieved by increasing the size of these components with respect to the operating wavelength, i.e., by overmoding these components. Similar attempts have been made to increase the power handling capabilities of bulk effect semiconductor components. Optically controlled semiconductor switches operating in overmoded waveguides have also been reported [6]. Here we report the development of a switch made from a $p-i-n$ diode array operating in an overmoded waveguide carrying the $\mathrm{TE}_{01}$ mode.

The $p-i-n$ diode $r f$ switch was invented in the middle of the 20th century $[7,8] . p-i-n$ diode switches have wide applications at the low to medium power levels. Various packaged $p-i-n$ diodes are commercially available in the market. However, due to their small size, packaged $p-i-n$ diodes cannot be used for switching very high power rf signals. Toward the end of the $1960 \mathrm{~s}$, a rectangular waveguide switch $[9,10]$ was developed to handle higher power rf signals than packaged $p-i-n$ diodes. The window switch was tested at the levels of $100 \mathrm{~kW}$ at $X$-band without problems. Thus, the power handling capability of a semiconductor rf switch has been at the levels of $100 \mathrm{~kW}$; it is still low for the applications to the active pulse compression systems for future linear colliders.

A high power device can comprise an array of active elements arranged together so that the total electromagnetic fields are reduced and the power handling capabilities are increased. In this paper, we will discuss high power $\mathrm{rf}$ switches which consist of the active switching elements. The basic idea is to distribute the power into several elements so that the amount of the power to be handled by each element is reduced, in addition to the improvement of the maximum power of each element, by designing it in an overmoded structure.

In Sec. II we present the design methodology of the high power rf switch and the scaling law that governs the relation between the number of elements and the power handling capability. The design and implementation of a $p-i-n / n-i-p$ diode array active window are described in Sec. III. We report on the results of low and high power experiments. We make some concluding remarks and point to future work in Sec. IV.

\section{DESIGN METHODOLOGY OF HIGH POWER MICROWAVE SWITCHES}

We will discuss the design methodology of high power microwave switches. We focus on SPDT (single pole double throw) switches, which have one input port and two output ports. Since one single active semiconductor device cannot handle very high power rf signals, the rf switch described here consists of several active switching elements. The load of the rf power is distributed to several elements; each element has to handle the reduced amount of the rf power.

\section{A. Synthesis of the rf switch}

The SPDT switch described here has two designs (see Fig. 1) [11-13]. Both designs consist of two $3 \mathrm{~dB}$ hybrids and two active modules. In the first design, the active module is an array of SPST (single pole single throw) 


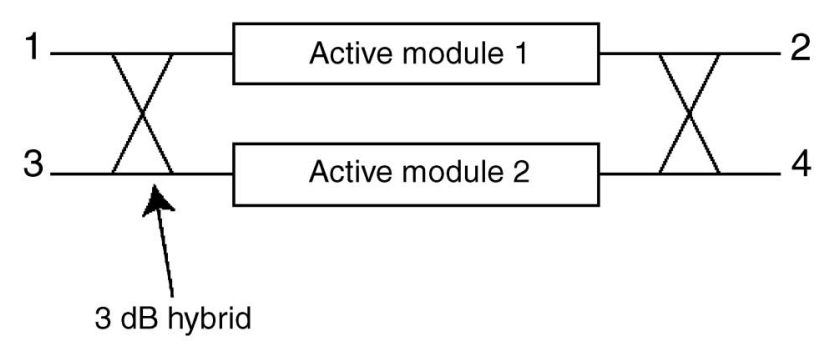

\section{Active module $=\left\{\begin{array}{l}\text { SPST array } \\ \text { Cascaded phase shifter }\end{array}\right.$}

FIG. 1. Schematic diagram of the switch system.

switches. In the second design, the module is composed of a cascaded phase shifter.

In the case of the SPST array (see Fig. 2), the active modules have two statuses, i.e., the perfect reflection status and the perfect transmission status. When an rf signal is reflected perfectly at the modules, all power goes to port 3. Also, when the modules transmit $100 \%$ of power, the output is port 4 .

In the case of the cascaded phase shifter (see Fig. 3), the phase difference between two active modules is chosen 0 and $\pi$. The output port is 4 and 2 with phase difference 0 and $\pi$, respectively.

Each cascaded element of the phase shifter and the SPST switch has a similar design: the active element. This element consists of a symmetrical three-port tee junction, a short plane in the third arm, and an active waveguide window placed in the third arm of the symmetrical tee junction. A schematic view of the active element is shown in Fig. 4.

The active window is a waveguide window that changes the reflection coefficient by the external control input.

3

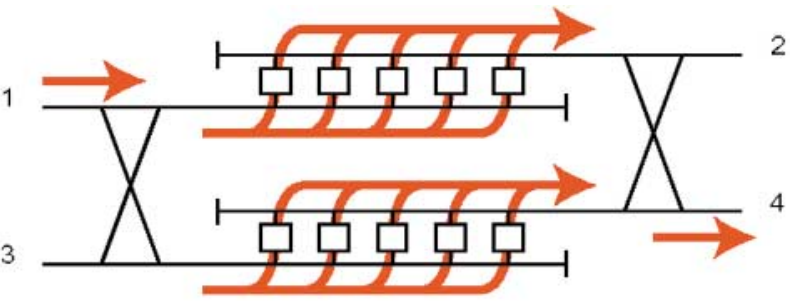

Active window off $/$ SPST $=$ perfect transmitter

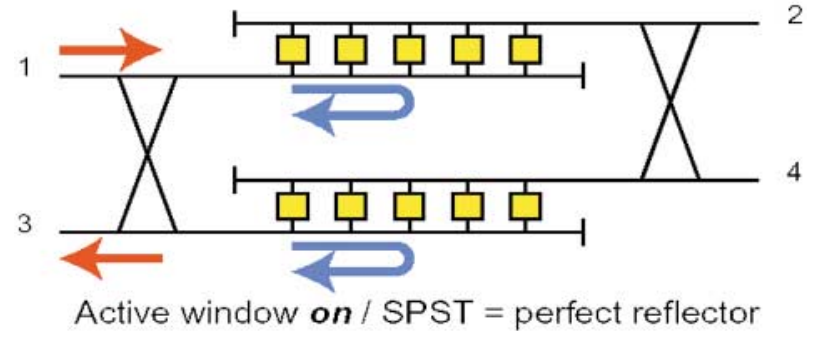

FIG. 2. (Color) Operating principle of SPST array.
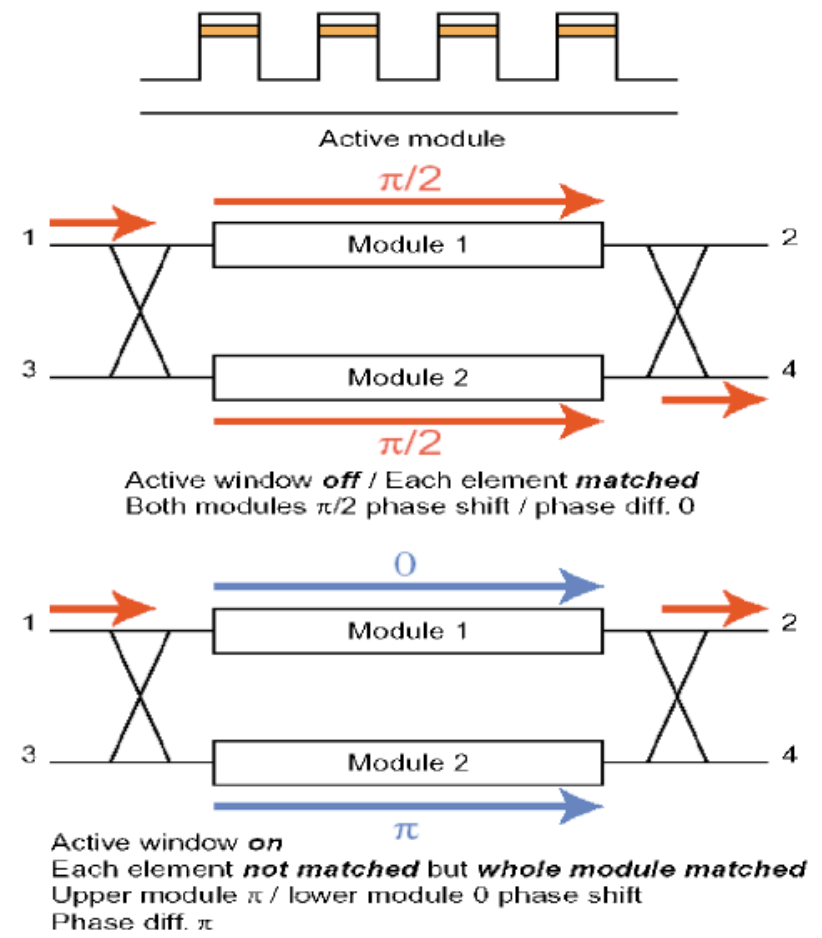

FIG. 3. (Color) Operating principle of cascaded phase shifter.

Here, the perfect transmission status of the active window is called off and the perfect reflection status is called on.

As a result of putting the short plane in the third arm, the active element is a two-port network. The basic idea of the active element is to change the $S$ matrix of the two-port network by changing the reflection phase in the third arm. As shown in Fig. 4, when the active window is on, the signal in the third arm is reflected at the active window. The situation is equivalent to putting a short plane at the position of the active window. Thus, the $S$ matrix of the two-port network changes.

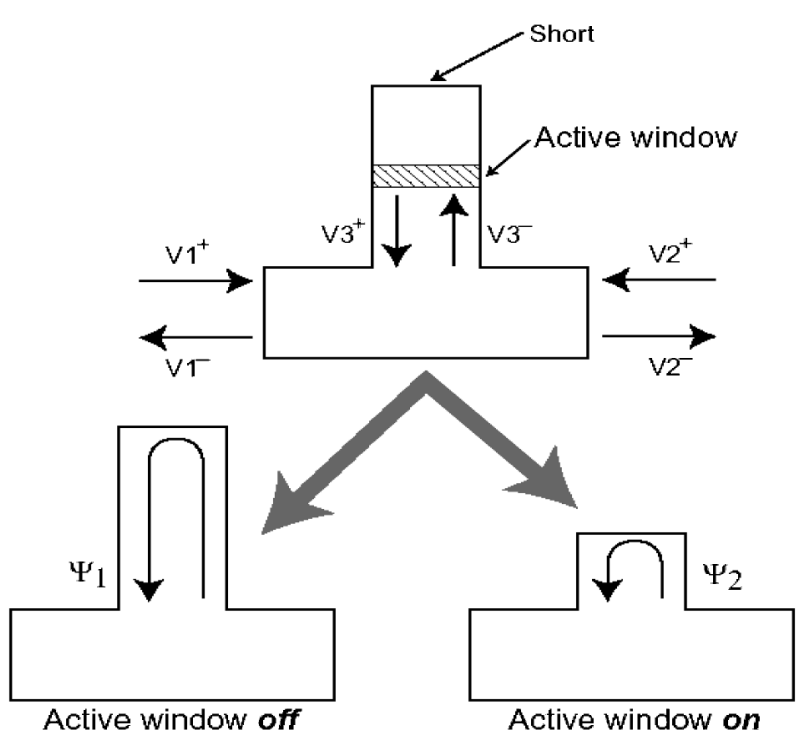

FIG. 4. The active element. 


\section{B. The three-port network as a control element}

A symmetrical tee junction of an arbitrary shape is illustrated in Fig. 5. The $S$ matrix of the lossless symmetrical tee junction may be parameterized [6] by defining an adequate reference plane. It is given by

$$
\underline{\underline{S}}=\left(\begin{array}{ccc}
\frac{e^{j \phi}-\cos \theta}{2} e^{j \alpha} & \frac{-e^{j \phi}-\cos \theta}{2} e^{j \alpha} & \frac{\sin \theta}{\sqrt{2}} e^{j \alpha} \\
\frac{-e^{j \phi}-\cos \theta}{2} e^{j \alpha} & \frac{e^{j \phi}-\cos \theta}{2} e^{j \alpha} & \frac{\sin \theta}{\sqrt{2}} e^{j \alpha} \\
\frac{\sin \theta}{\sqrt{2}} e^{j \alpha} & \frac{\sin \theta}{\sqrt{2}} e^{j \alpha} & \cos \theta
\end{array}\right)
$$

where the parameters $\phi$ and $\theta$ determine the coupling between the ports of the tee junction, and $\alpha$ is a parameter which specifies the location of the reference planes of ports

$$
\underline{\underline{S}}=\left(\begin{array}{cc}
\cos \left(\frac{\zeta-\phi}{2}\right) e^{j[(\phi / 2)+(\zeta / 2)+\alpha]} & j \sin \left(\frac{\zeta-\phi}{2}\right) e^{j[(\phi / 2)+(\zeta / 2)+\alpha]} \\
j \sin \left(\frac{\zeta-\phi}{2}\right) e^{j[(\phi / 2)+(\zeta / 2)+\alpha]} & \cos \left(\frac{\zeta-\phi}{2}\right) e^{j[(\phi / 2)+(\zeta / 2)+\alpha]}
\end{array}\right),
$$

where $\zeta$ is defined by

$$
e^{j \zeta} \equiv \frac{\cos \theta-e^{j \psi}}{\cos \theta e^{j \psi}-1} .
$$

Since the tee junction is a lossless network, the wave in the third arm is a pure standing wave. The maximum standing wave voltage in the third arm at the distance $\xi$ from the short plane is obtained as

$$
\left|V_{3}\right|=\left|V_{3}^{-}\right|\left|1-e^{2 j \beta_{3} \xi}\right|=2\left|V_{3}^{-}\right|\left|\sin \beta_{3} \xi\right| .
$$

The amplitude of the normalized voltage in the third arm is given by

$$
\left|V_{3}^{+}\right|=\left|V_{3}^{-}\right|=\frac{1+\cos ^{2} \theta+2 \cos \theta \cos \zeta}{2 \sin ^{2} \theta}\left|V_{1}^{+}+V_{2}^{+}\right| .
$$

Hence, the maximum electric field of the standing wave in the third $\operatorname{arm} E_{\max }$ at the distance $\xi$ from the short plane is given by

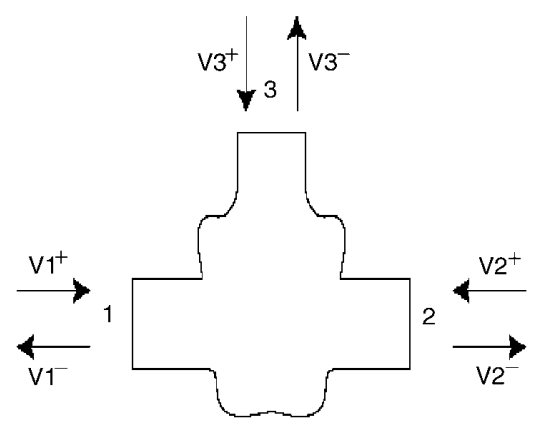

1 and 2. The scattered rf signals $\underline{V}^{-}$are related to the incident rf signals $V^{+}$by $\underline{V}^{-}=\underline{S V^{+}}$, where $V_{i}^{ \pm}$represents the incident/reflected rf signal from the $i$ th port.

When a short plane terminates the third arm, the wave reflected at the short plane has the same amplitude, and has the phase advance $\Psi$, which is determined by the distance $d$ between the reference plane and the short plane. The voltage of the reflected wave is given by

$$
V_{3}^{+}=V_{3}^{-} e^{2 j \beta_{3} d}=V_{3}^{-} e^{j \Psi},
$$

where $\beta_{3}$ is the wave number in the third arm and $\Psi$ is the reflection phase advance. The scattering matrix parameters for the symmetric two-port network formed by the shorted three-port network can be calculated from Eqs. (1) and (2) and are given by

$$
\begin{aligned}
E_{\max }= & 2\left(\frac{1+\cos ^{2} \theta+2 \cos \theta \cos \zeta}{2 \sin ^{2} \theta}\right)^{1 / 2} \\
& \times\left|\sin \beta_{3} \xi\right|\left(\frac{P_{\mathrm{in}} Z}{A G}\right)^{1 / 2},
\end{aligned}
$$

where $P_{\text {in }}$ is the input power which is equal to $\mid V_{1}^{+}+$ $V_{2}^{+} I^{2}, Z_{g}$ is the waveguide impedance, $A$ is the cross sectional area of the third arm waveguide, and $G$ is a geometrical factor that depends on the mode and the waveguide shape of the third arm.

When the short plane has a surface resistivity $R_{s}$, the loss $P_{l}$ dissipated into the short plane is given by $4\left|V_{3}^{-}\right|^{2} \frac{R_{s}}{Z_{g}}$. Using Eq. (6) the loss dissipated into the short plane in the third arm is explicitly given by

$$
P_{l}=4 \frac{1+2 \cos \theta \cos \zeta+\cos ^{2} \theta}{2 \sin ^{2} \theta} \frac{R_{s}}{Z_{g}} P_{\text {in }} .
$$

\section{SPST array}

The operating principle of the SPST array is shown in Fig. 2. In the SPST array configuration, the $3 \mathrm{~dB}$ hybrid

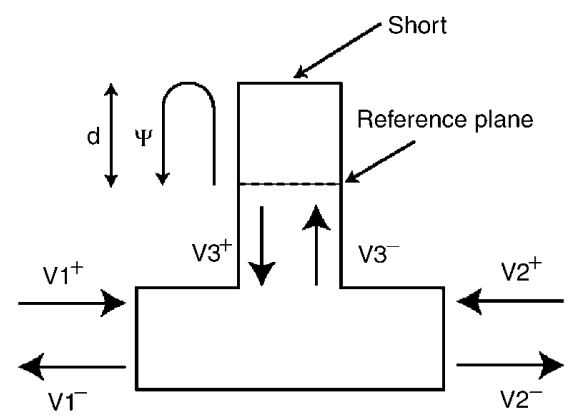

FIG. 5. (a) Symmetrical tee-junction element. (b) The three-port network as a control element. 
and the power dividers divide the input power, then the power is guided to each SPST switch. The SPST switches described here work as a perfect transmitter and a perfect reflector when the active windows are off and on, respectively. When the active windows are off, each divided power goes through the SPST switches and is combined in the power divider. The recombined power goes to port 4 through the $3 \mathrm{~dB}$ hybrid. Also, when the active windows are on, each SPST switch reflects the power perfectly, and then all power goes to port 3 .

From the $S$ matrix of the two-port network Eq. (3), the matching condition of the SPST switches, i.e., $S_{11}=0$, is given by

$$
\zeta_{0}=\phi+(2 N+1) \pi,
$$

where $N$ is an integer. Hence, the short plane is placed at the position where $\Psi_{0}$ satisfies the following condition:

$$
e^{j \Psi_{0}}=\frac{e^{j \phi}-\cos \theta}{-1+e^{j \phi} \cos \theta} .
$$

Also, the perfect reflector condition, i.e., $S_{11}=1$, is given by

$$
\zeta_{1}=\phi+2 N \pi
$$

In this case, the active window is placed at the position where $\Psi_{1}$ satisfies the following condition:

$$
e^{j \Psi_{1}}=\frac{e^{j \zeta_{1}}+\cos \theta}{1+e^{j \zeta_{1}} \cos \theta} .
$$

In the SPST array configuration, each active element handles $1 / n$ of the total input power, where $n$ is the number of elements. The maximum electric field at the active window when the active window is off and $\zeta_{0}=\phi+\pi$ is given by

$$
\begin{aligned}
E_{\max }= & 2\left(\frac{1+\cos ^{2} \theta-2 \cos \phi \cos \theta}{n \sin ^{2} \theta}\right)^{1 / 2} \\
& \times\left|\sin \frac{\Delta \psi}{2}\right|\left(\frac{P_{\text {in }} Z_{3}}{A_{3} G_{3}}\right)^{1 / 2},
\end{aligned}
$$

where $\Delta \psi=\psi_{0}-\psi_{1}$ and can be calculated from Eqs. (10) and (12), and the suffix 3 in the last term refers to quantities that define the properties of the third arm and have the same definitions as in Eq. (7).

The total loss $P_{l}$ dissipated into the active windows when the active window is on and $\zeta_{1}=\phi$ is given by

$$
P_{l}=2 \frac{1+\cos ^{2} \theta+2 \cos \phi \cos \theta}{\sin ^{2} \theta} \frac{R_{s}}{Z_{3}} P_{\text {in }} .
$$

\section{Cascaded phase shifter}

The operating principle of the cascaded phase shifter is illustrated in Fig. 3. In the case of the cascaded phase shifter, the active modules have two states. The phase difference between the two active modules is 0 or $\pi$. We may choose the total phase shift of the modules as follows: (i) The total phase shift in each of the upper and lower active modules in Fig. 3 is $\pi / 2$. The phase difference between the two modules is 0 . The output port is port 4 in Fig. 3. This status is realized when the active windows in the active elements are off and each element is matched by itself. (ii) Total phase shift in each of the upper and lower active modules is 0 and $\pi$, respectively. The phase difference between the two modules is $\pi$. The output port is port 2. This status is realized when the active windows in the active elements are on. Each element is not matched by itself, but the whole module is matched by cascading.

In the status of phase difference 0 , each active element is set to be matched by itself. The matching condition of the active elements is $\zeta_{0}=\phi+\pi$, the phase $\Psi_{0}$ is given by Eq. (10).

Substituting into Eq. (3), we find that the phase advance of each element is $\phi+\alpha+\pi$. Each active module consists of $n / 2$ elements and has the total phase shift $\pi / 2$. The total number of elements in two modules is $n$. This means $(\phi+\alpha+\pi) n / 2=\pi / 2$; that is, $\alpha=$ $\pi / n-\pi-\phi$. The reference plane of ports 1 and 2 of the active element is chosen so that this condition is satisfied.

When the active window is on, each element is not matched by itself, but the whole module is matched. The matching condition is given by following the procedure.

We may introduce the transfer matrix $A$ of a two-port network [11,14]. Each active module consists of $n / 2$ active elements. When the active module is matched and the total phase shift across the module is $\pi$, the transfer matrix must satisfy $A^{n / 2}=-I$, where $I$ is the identity matrix. Hence, the eigenvalues of $A$ are $e^{ \pm j[(4 m+2) \pi / n]}$, where $m$ is an integer. Similarly, when the phase shift of the module is 0 , the transfer matrix satisfies $A^{n / 2}=I$ with eigenvalues given by $e^{ \pm j[(4 m+4) \pi / n]}$.

From these eigenvalues of the transfer matrix, we can derive the condition of $\zeta_{I}$. Explicitly,

$$
\zeta_{1}=\phi-2 \tan ^{-1}\left(\frac{\sin \frac{\pi}{n}}{\cos \frac{\pi}{n}-\cos \frac{M \pi}{n}}\right),
$$

where $M$ is an integer given by

$$
M= \begin{cases}4 m+4, & \text { total phase shift is } 0, \\ 4 m+2, & \text { total phase shift is } \pi .\end{cases}
$$

By noting that each active window handles one-half of the total input power, the maximum field at the active window when it is off can be explicitly calculated,

$$
\begin{aligned}
E_{\max }= & \left(\frac{1+\cos ^{2} \theta-2 \cos \phi \cos \theta}{\sin ^{2} \theta}\right)^{1 / 2} \\
& \times\left|\sin \frac{\Delta \psi}{2}\right|\left(\frac{P_{\text {in }} Z_{3}}{A_{3} G_{3}}\right)^{1 / 2},
\end{aligned}
$$

where $\Delta \psi=\psi_{0}-\psi_{1}$ and can be calculated from Eqs. (10) and (12) with the appropriate value for $\zeta_{1}$ given by Eq. (15). Note that with increasing the number of elements $n$, the phase $\Delta \psi$ becomes smaller, hence, the maximum voltage at the window is reduced. 
The loss dissipated into the active window in each active element when the active window is on is given by

$$
P_{l}(\text { each })=2 \frac{1+\cos ^{2} \theta+2 \cos \phi \cos \theta}{\sin ^{2} \theta} \frac{R_{s}}{Z_{3}}\left|V_{1}^{+}+V_{2}^{+}\right|^{2} .
$$

Since each element is not matched by itself, there is a standing wave in the waveguides between the elements. Thus, the normalized voltage $\left|V_{1}^{+}+V_{2}^{+}\right|$when the active window is on is always more than 1 and it depends on the position of the element in the module. With increasing the number of elements, $\left|V_{1}^{+}+V_{2}^{+}\right|$becomes closer to the minimum value, near 1 . Assuming the number of elements is large enough and $\left|V_{1}^{+}+V_{2}^{+}\right|$is near 1 , the total loss dissipated into the active windows is approximated by

$$
P_{l}(\text { total })=\frac{n\left(1+\cos ^{2} \theta+2 \cos \phi \cos \theta\right)}{\sin ^{2} \theta} \frac{R_{s}}{Z_{3}} P_{\text {in }} .
$$

\section{E. Scaling law}

We will discuss the scaling law, which governs the relation between the power handling capability and the number of active elements. Since we are interested in switching rf signals at very high power levels, it is important to estimate the number of elements required to handle a certain amount of power. The criteria that determine the number of elements are (i) the maximum electric field at the active window must be kept below the maximum electric field limit of the semiconductor device and (ii) the loss dissipated into the active window must be kept within a priory given value that depend on the application. On most $\mathrm{rf}$ pulse compression systems the switch is required to have losses below $2 \%$.

We have derived expressions for the maximum electric field at the active window when the active window is off, and the loss dissipated into the active window when the active window is on, in the cases of both the SPST array and the cascaded phase shifter. By relating these expressions, we will derive the scaling law. First we notice that the scattering matrix $S$ for the three-port network contains two variables, $\theta$ and $\phi$. Indeed, for any choice of $\phi$, one can find a value for $\theta$ such that Eqs. (13), (14), (17), and (19) preserve their values. Hence the choice of the angle $\phi$ is arbitrary. For simplicity we will set $\phi=0$.

Let us start with the case of the SPST array. We assume that the active element is matched by itself when the active window is off, and it is a perfect reflector when the active window is on. Let us define $L_{0}$ as the acceptable relative loss determined from external requirements Then, at the limit, $P_{l}$ (total) $/ P_{\text {in }}=L_{0}$. With the previous assumption about $\phi$, one can write

$$
L_{0} \equiv P_{l}(\text { total }) / P_{\text {in }}=2 \frac{R_{s}}{Z_{g}} \tan ^{2} \frac{\theta}{2},
$$

where $R_{s}$ is the surface resistance and $Z_{g}$ is the waveguide impedance in the third arm. Using the above equation one can write Eq. (13) as

$$
E_{\max }=2\left(\frac{R_{s} P_{\text {in }}}{n L_{0} A G}\right)^{1 / 2} .
$$

Similarly, for the cascaded phase shifter one can relate the losses to the peak field. In the case of the cascaded phase shifter, the active element is matched by itself when the active window is off and the total change of the phase advance of the active module is $\pm \pi / 2$. We assume that the total number of active elements $n$ is large enough so that the phase shift of each element when the active window is on is fairly small. With this assumption, the reflection phase change $\Delta \psi \ll 1$ and it would not disturb the matching condition. Also, we can neglect the effect of the standing waves between each active element. With these assumptions one can show that the losses are given by

$$
L_{0}=\frac{2 \pi R_{S}}{\Delta \psi Z_{g}} .
$$

With the help of this equation and the above assumptions, Eq. (17) becomes

$$
E_{\max }=\pi\left(\frac{R_{s} P_{\text {in }}}{n L_{0} A G}\right)^{1 / 2}
$$

From Eqs. (21) and (23) one can write the scaling law for both the SPST array and the cascaded phase shifter array,

$$
n=C^{2} \frac{P_{\text {in }}}{L_{0}} \frac{1}{A G} \frac{R_{s}}{E_{\text {max }}^{2}},
$$

where $P_{\text {in }}$ is the total input power, $R_{S}$ is the surface resistance of the active window when it is on, $L_{0}$ is the acceptable relative loss determined by external requirements, $n$ is the total number of active elements, $A$ is the cross sectional area of the waveguide, and $G$ is a geometrical factor that depends on the mode and the shape of the waveguide. In the above equation, $C$ is a constant, $\pi$ for the cascaded phase shifter and 2 for the SPST switch array. This means that the case of the SPST array is better than that of the cascaded phase shifter. However, the case of the cascaded phase shifter might offer an easier implementation since it does not require the set of splitters to distribute the power as in the SPST array case.

It is now appropriate to make some remarks about this scaling law. (i) The SPST array configuration needs less active elements than the cascaded phase shifter. (ii) The number of elements is proportional to the surface resistance, and inversely proportional to the acceptable loss. Hence, we should employ the active window with low loss when the window is on. (iii) The number of elements is inversely proportional to the square of the peak electric field limit. Thus, it is necessary to choose the material and the device which can endure high electric fields, as the active window. (iv) The number of elements is independent of the waveguide impedance $Z_{g}$. The effects of $Z_{g}$ on the 


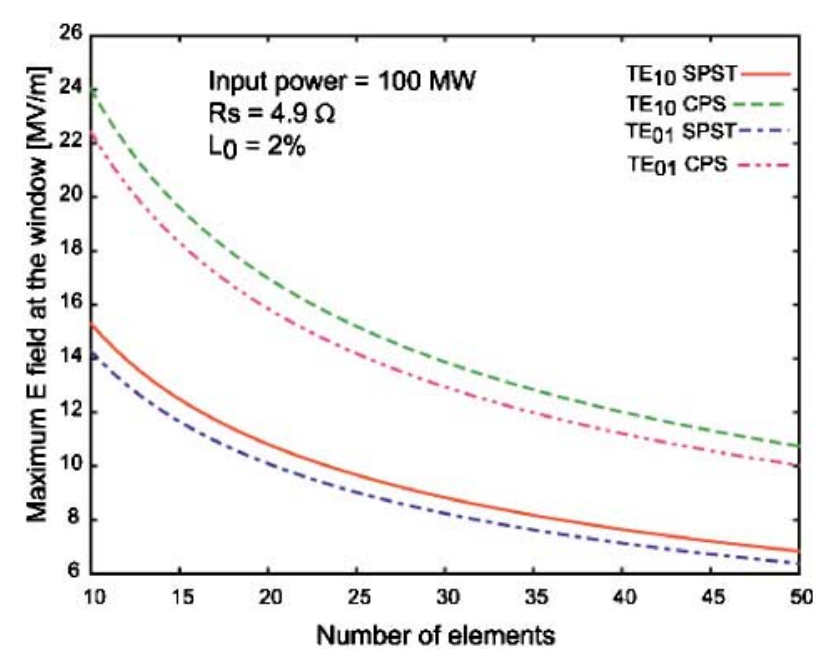

FIG. 6. (Color) Number of elements versus maximum electric field of the active window.

increase of electric field and the decrease of loss cancel each other.

In the derivation of the scaling law, we did not use any device-specific characteristics. Hence, this scaling law is quite general, it can be used not only for the $p-i-n / n-i-p$ diode switches described in the following sections, but also for the optically driven switches [6] or other kinds of active switches, such as magnetic switches [5].

Now we can estimate the required number of elements for a given application. For example, we use the $p-i-n / n-i-p$ diode array active window, described in the following sections, with the following assumptions: (i) the operating frequency is $11.424 \mathrm{GHz}$; (ii) the maximum field limit on the silicon window is $10 \mathrm{MV} / \mathrm{m} \mathrm{[7];}$ (iii) the acceptable loss $L_{0}$ when the active window is on is $2 \%$, which is required for the application to the pulse compression systems [3]; (iv) the surface resistance $R_{S}$ obtained by a maximum carrier density of $10^{17} \mathrm{~cm}^{-3}$ is $4.9 \Omega$; and (v) the total input power is $100 \mathrm{MW}$.

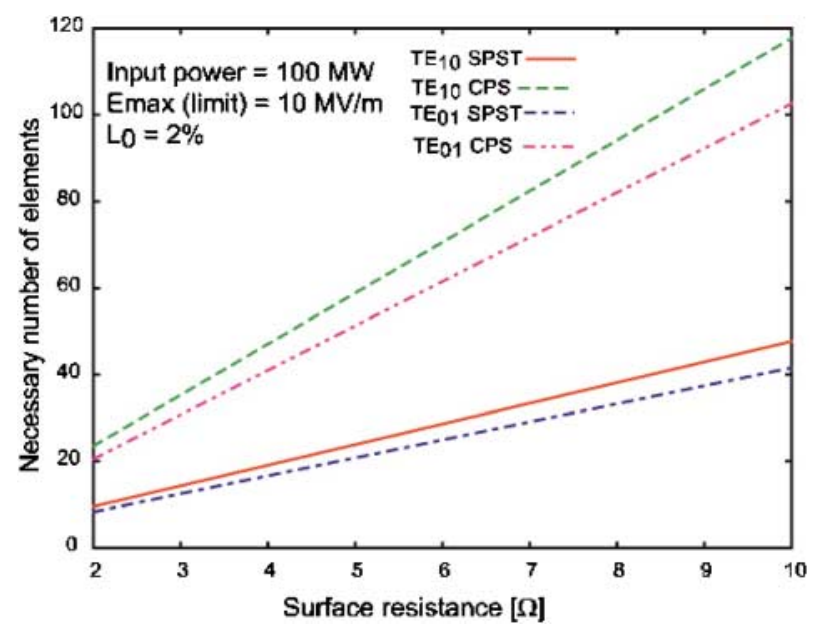

FIG. 7. (Color) Surface resistance versus necessary number of elements.

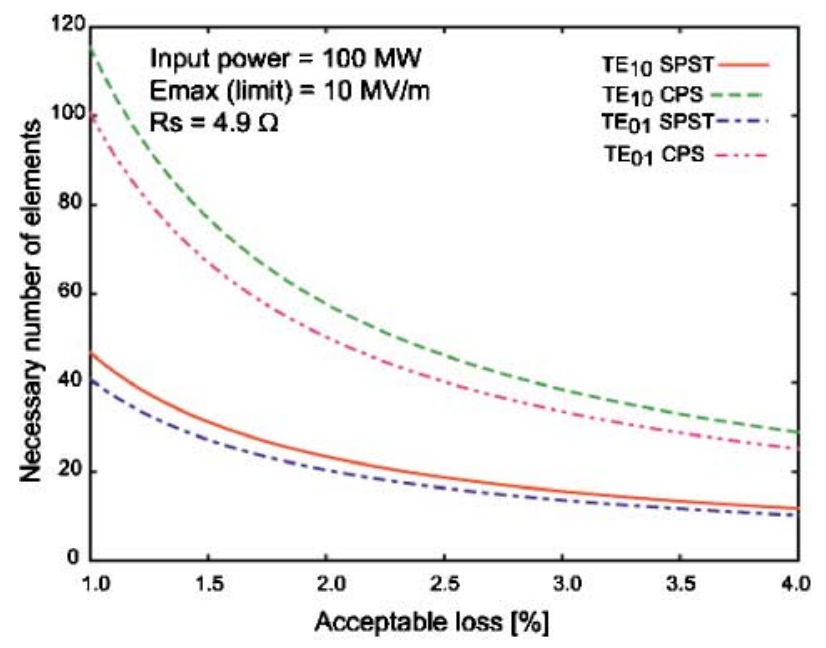

FIG. 8. (Color) Acceptable loss versus necessary number of elements.

If we employ $\mathrm{TE}_{10}$ mode active windows in WR90 rectangular waveguides, we find from Eq. (24) that 24 and 58 elements are needed for the SPST array and the cascaded phase shifter, respectively. With $\mathrm{TE}_{01}$ mode active windows in 1.3-in. diameter circular waveguides, 21 and 51 elements are needed, respectively. The correlation between the number of elements and the maximum electric field at the active window is shown in Fig. 6. In the figure, the input power, the acceptable loss, and the surface resistance are fixed. From the figure, we can clearly see that we must employ the material and the device that has a high field limit as the active window to save the number of elements. Also, in Figs. 7 and 8 the correlations between the number of elements and the parameters concerning the loss at the window, the surface resistance, and the acceptable loss are shown. As described above, the number of elements is proportional to the surface resistance when the active window is on; we must choose the window that has a high conductivity when the window is on. If the external requirements allow a larger loss for the total system, the number of elements becomes less. However, the local heating at the window will be an issue. Independent of the scaling law, this issue must be handled carefully.

\section{DESIGN AND IMPLEMENTATION OF $p-i-n / n-i-p$ DIODE ARRAY ACTIVE WINDOW}

A new active window, $p-i-n / n-i-p$ diode array active window which is operated at $\mathrm{TE}_{01}$ mode in the circular waveguide was proposed and developed. Our active window is designed and built to avoid the difficulties of the $\mathrm{TE}_{10}$ mode rectangular waveguide window switch and handle $X$-band rf signals at multimegawatt levels. This is achieved by using an overmoded structure hence increasing the cross sectional area and reducing the power density. Since the $\mathrm{TE}_{01}$ mode in circular waveguide does not have any electric field at the waveguide wall, this makes the design more robust by avoiding edge effects. 
In this section, the design of our $p-i-n / n-i-p$ diode array active window will be described.

\section{A. The silicon window}

The conceptual view of our active window is illustrated in Fig. 9. The base material of the window is highly pure silicon. This window is located in a circular waveguide, which is operated at the $\mathrm{TE}_{01}$ mode. As shown in Fig. 9, the $p-i-n / n-i-p$ diode structure is a set of radial lines. Each line is heavily doped by $p$-type and $n$-type impurities on the topside and back side surfaces, respectively. Each diode line is covered by a metal line, which supplies bias voltage and current to the diode line. The electric field of $\mathrm{TE}_{01}$ is shown in Fig. 9. All electric field lines of the $\mathrm{TE}_{01}$ mode are in the azimuthal direction; i.e., the diode lines are perpendicular to the electric field of the rf signal. This means that the reflection caused by the diode structure and the metal lines is very small when the active window is reverse or zero biased; the rf signal sees only the dielectric contribution of the bulk silicon material. This is the off status of the active window. To minimize the reflection from the lines, the coverage factor, which is defined as the ratio of the area of the diode structure to that of the whole active region of the window, is chosen to be $10 \%$. The $p$ lines on the topside and the $n$ lines on the back side are arranged to alternate with each other (see Fig. 9). A side-cut view of the active window illustrates the idea; see Fig. 10. When forward bias is applied, a massive number of injected carriers go across the $i$ region. Since the $p$ and $n$ lines are alternating, the injected carriers fill the $i$ region; the incident rf signal is reflected. This is the on status of the active window. The thickness of the active window, which is the same as the $i$ region width in this design, must be small enough so that the carriers injected from the heavily doped $p$ and $n$ lines by the forward bias can diffuse through the high receptivity $i$ region to the heavily doped lines on the other side. This is

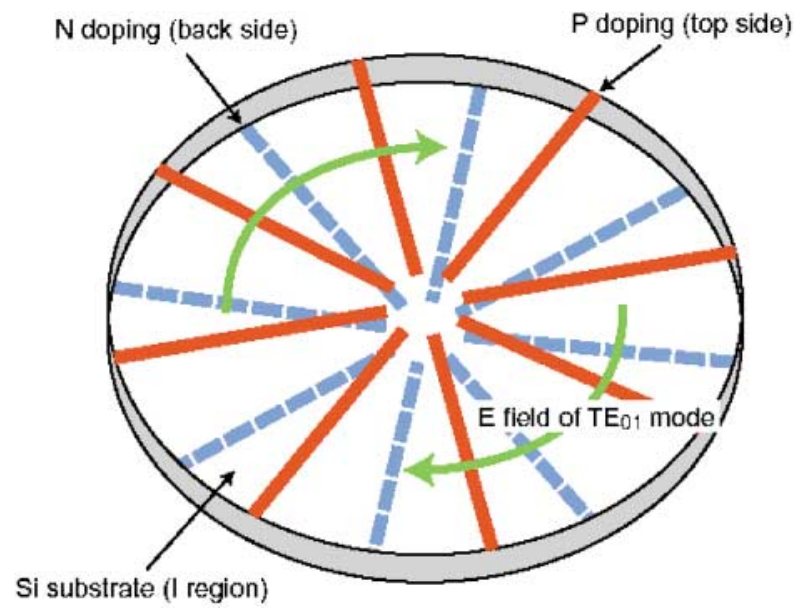

FIG. 9. (Color) Conceptual view of $p-i-n / n-i-p$ diode active window.

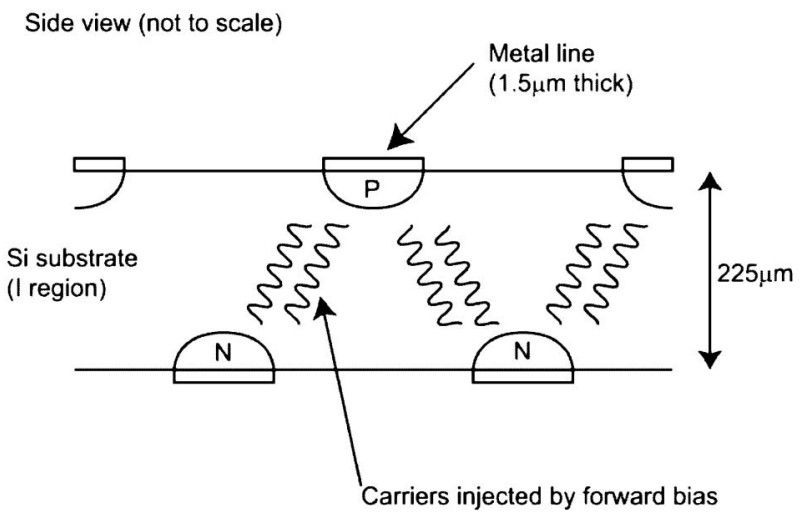

FIG. 10. Side view of $p-i-n / n-i-p$ diode active window.

a very important point to achieve good rf isolation at the on status. The carrier lifetime in the high resistivity silicon material is closely related to the diffusion length of the carriers. Namely, $L_{D}=\left(\tau D_{A P}\right)^{1 / 2}$, where $L_{D}$ is the diffusion length of the carriers, $\tau$ is the carrier lifetime in the silicon material, and $D_{A P}$ is the diffusion coefficient. The diffusion coefficient in silicon is $D_{A P}=15.6 \mathrm{~cm}^{2} / \mathrm{s}$, then we get $L_{D} \approx 40[\tau(\mu \mathrm{sec})]^{1 / 2} \mu \mathrm{m}$. Hence, the base material of the active window must be very pure silicon and must have long carrier lifetime to achieve good conductivity modulation with forward bias. While this puts an upper limit on the window thickness, there is a lower limit for this thickness. If the window is much thinner than the skin depth, good isolation of the rf signal would not be obtained. If we assume the carrier density to be $10^{17} \mathrm{~cm}^{-3}$ in the $i$ region with forward bias, then the skin depth $\delta_{s}$ can be calculated by the knowledge of the carrier motilities of silicon. At a frequency of $11.424 \mathrm{GHz}, \delta_{s} \approx 100 \mu \mathrm{m}$.

In the actual design of our active window, the diode structure consists of 400 radial lines each on the topside and the back side. The width of the radial lines are tapered, it is $25 \mu \mathrm{m}$ near the waveguide surface and $2 \mu \mathrm{m}$ near center of the active window. This line width and the number of lines are chosen so that the coverage factor is $10 \%$. This active window is operated at $11.424 \mathrm{GHz}$. The diameter of the active region of the window is $1.299 \mathrm{in}$. Also, the thickness of the window is $225 \mu \mathrm{m}$. If the carrier density achieved $10^{17} \mathrm{~cm}^{-3}$, this thickness is more than the skin depth of the intrinsic region for $X$-band rf signals.

At a carrier density of $10^{17} \mathrm{~cm}^{-3}$ the surface resistance $R_{s} \approx 4.8 \Omega$ at $11.424 \mathrm{GHz}$. In our design, the diameter of the active region and waveguide is 1.299 in., so that the waveguide impedance is 4.16 times the impedance of vacuum. Hence, the loss dissipated into the window, when the window is on, is given by $L_{0} \equiv P_{l}($ total $) / P_{\text {in }}=$ $4 \frac{R_{s}}{Z_{g}} \approx 1.23 \%$.

\section{B. Process flow}

The processing of the $p-i-n / n-i-p$ diode array active windows was done by the author at the Stanford 
Nanofabrication Facility [15] of Stanford University. As shown in the previous section, the carrier lifetime is strongly related to the carrier density in the intrinsic region when forward bias is applied. Since the $i$ region width is long [the same as the wafer thickness $(225 \mu \mathrm{m})$ ], a pure material with very high resistivity is needed to achieve high density of the carriers injected by forward bias. Our starting material, silicon wafers, is doped approximately $10^{12} \mathrm{~cm}^{-3}$, and the resistivity is more than $5000 \Omega \mathrm{cm}$. The minority carrier lifetime in the material is more than a millisecond.

The complete process flow is shown in Table I. The process of the $p-i-n / n-i-p$ diode structure is based on a common and standard integrated circuit (IC) fabrication process. The method is illustrated in Fig. 11. The initial oxidation process makes the silicon dioxide layers on the surfaces of the wafers. The target thickness of the oxide layer is $4000 \AA$. The oxide thickness is checked by the noncontact spectroreflectometry measurement. Boron and phosphorus are chosen as the $p$-type and $n$-type impurities, respectively. They are implanted at $100 \mathrm{keV}$ into the silicon substrate through the holes of the oxide layer. These holes of the oxide layer are formed by the wet etching process in hydrofluoric acid.

The aligner manufactured by Karl Suss used to define the lines and the holes is a contact aligner and has resolution better than $0.75 \mu \mathrm{m}$ for both topside and back side alignments; this aligner is accurate enough to define our diode structures. The dose shown in the table is for the $10^{16} \mathrm{~cm}^{-3}$ version of the doping density. For the $10^{17} \mathrm{~cm}^{-3}$ version, 10 times heavier dose is used. After the implantation of the impurities, the high temperature drive-in process is done to diffuse the impurities into the
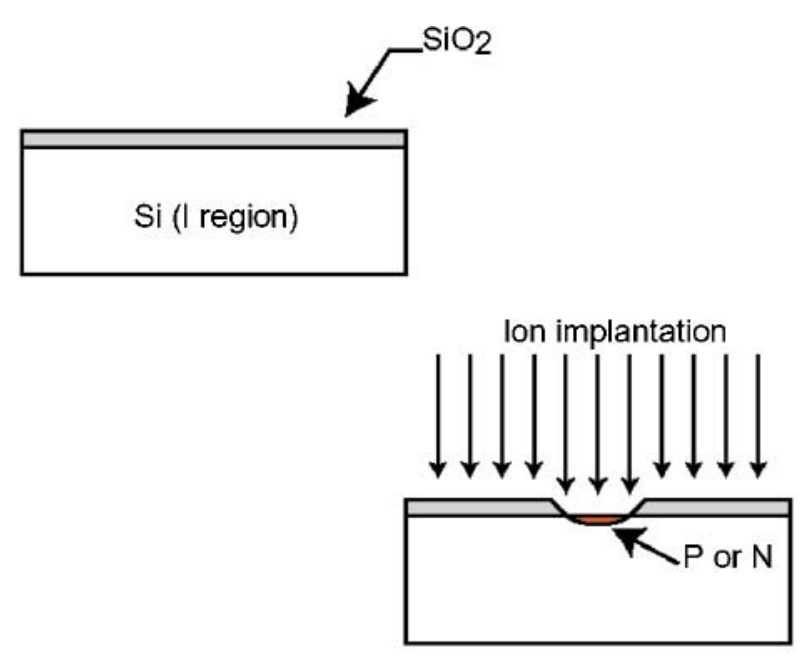

After drive-in and metallization

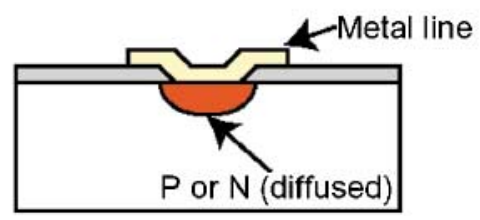

FIG. 11. (Color) Fabrication method.

silicon substrate. The depth of the diffusion is about $5 \mu \mathrm{m}$ after $16 \mathrm{~h}$ of the drive-in process at $1150{ }^{\circ} \mathrm{C}$. In the same process, a $2000 \AA$ thick oxide layer is formed on the hole used for the implantation of the impurities.

Then, the contact holes are formed over the diffusion lines by a wet etching process so that the metal lines and

TABLE I. Process flow for $p-i-n / n-i-p$ diode array active window.

\begin{tabular}{|c|c|}
\hline Mask & Purpose and parameters \\
\hline & Starting material: $\langle 100\rangle n$-type $5000-\mathrm{cm} 4$ in. Si wafers \\
\hline & Initial oxide growth $0.4 \mu \mathrm{m}$ in steam at $1000{ }^{\circ} \mathrm{C}$ for $1 \mathrm{~h}$ \\
\hline \multirow{3}{*}{ Mask 1 (top) } & $p$-doping definition \\
\hline & Oxide etch topside \\
\hline & Implant topside: boron $100 \mathrm{keV} 6 \times 10^{13} \mathrm{~cm}^{-2}$ \\
\hline \multirow[t]{5}{*}{ Mask 1 (back) } & $n$-doping definition back side alignment done by Karl Suss aligner \\
\hline & Back side alignment done by Karl Suss aligner \\
\hline & Oxide etch back side \\
\hline & Implant back side: phosphorus $100 \mathrm{KeV} 6 \times 10^{13} \mathrm{~cm}^{-2}$ \\
\hline & $\begin{array}{l}\text { Drive-in impurities into silicon: at } 1000^{\circ} \mathrm{C}: 35 \mathrm{~min} \text { Ar, } 10 \mathrm{~min} \text { dry } \mathrm{O}_{2}, 30 \text { min wet } \mathrm{O}_{2} \text {, } \\
10 \mathrm{~min} \text { dry } \mathrm{O}_{2} \text {, at } 1150^{\circ} \mathrm{C}: 16 \mathrm{~h}\end{array}$ \\
\hline \multirow[t]{3}{*}{ Mask 2 (top and back) } & Contact hole definition \\
\hline & Oxide etch both sides \\
\hline & Metal deposition (both sides), sputter aluminum $1.5 \mu \mathrm{m}$ thick \\
\hline \multirow[t]{2}{*}{ Mask 3 (top and back) } & Metal line definition \\
\hline & Etch aluminum (wet etch) \\
\hline \multirow[t]{4}{*}{ Mask 4 (top) } & Deep trench etch definition \\
\hline & Bond backing support wafers \\
\hline & Deep silicon etch thorough wafers $(225 \mu \mathrm{m})$ by Surface Technology Systems deep reactive ion etcher \\
\hline & Remove backing wafer by dipping in acetone (several days) \\
\hline
\end{tabular}


the diffusion lines contact each other through the contact hole. Aluminum is sputtered on the surfaces of the wafers. The thickness is set to be $1.5 \mu \mathrm{m}$. The metal line is formed through the wet etching process by mixed acid. Finally, a deep trench etched at the design diameter of the window cuts the wafer.

\section{C. rf structures and window support}

The rf structure to support the window is shown in Fig. 12. It consists of two aluminum circular waveguides with steps, a ceramic ring, and metal springs. The active window is located between the two waveguides. The ceramic ring fixes the active window at the design position.

The $\mathrm{TE}_{01}$ mode $\mathrm{rf}$ signal is launched by a compact wrap-around mode converter [16]. The diameter of the waveguides changes from $1.5 \mathrm{in}$. at the low impedance section to $1.3 \mathrm{in}$. at the high impedance section by the steps. The steps are designed so that the whole rf structure is matched by itself without the active window. Since silicon has a large dielectric constant, the impedance mismatch at the surface of the active window is large; the reflection from the window surface caused by this mismatch is not zero. However, this rf structure is designed and built without a matching section, which compensates for the mismatch at the surfaces of the active window. This is done because we are interested in the pure characteristics of the active window.

The two waveguides are dc separated. The ceramic ring works as the dc voltage gap. There are metal springs between the active window and the waveguides. The waveguides are connected to a biasing circuit. The biasing voltage is supplied to the active window through the waveguides and the springs. The waveguides are dc isolated from the $\mathrm{TE}_{01}$ mode converters by a Mylar insulators.

There is a gap between the two waveguides, but no choke structure. Since the surface currents of $\mathrm{TE}_{01}$ mode

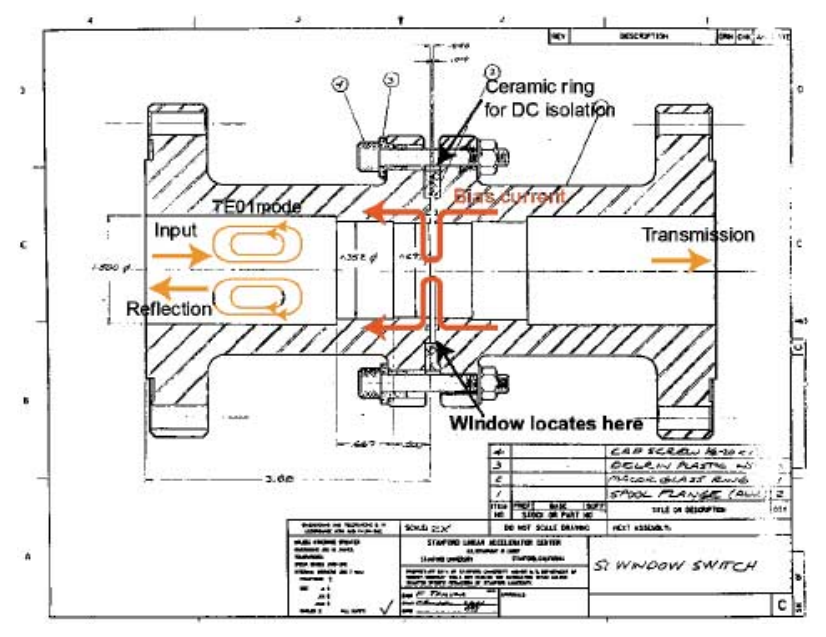

FIG. 12. (Color) Active window and rf structure. in circular waveguides are azimuthal, the gap does not cut any surface currents and there is no rf leakage through the gap. Indeed, this a big advantage of this structure over $\mathrm{TE}_{10}$ mode in rectangular window switches. We can avoid the complex choke structure, which is necessary in the rf structures of this later type.

Finally, since this structure needs to operate under vacuum, all gaskets for sealing the vacuum are Viton rubber gaskets for $\mathrm{dc}$ isolation.

\section{EXPERIMENTAL RESULTS}

\section{A. Low power measurements}

Two types of low power measurements were performed: the network analyzer measurement and the active switching experiment with biasing. In the network analyzer measurement, the passive characteristics of the window supporting rf structure and the active window were examined. Also, in order to test the basic switching performance of the $p-i-n / n-i-p$ diode array active window, the active switching experiment with a low power rf source was performed.

The schematic diagram of the passive measurement is shown in Fig. 13. The $S$ parameters $\left(S_{11}\right.$ and $\left.S_{21}\right)$ of test devices, the window supporting rf structure, and the $p-i-n / n-i-p$ diode array active window are measured by the network analyzer (HP8510) at the operating frequency $11.424 \mathrm{GHz}$.

Without the window, the $S$ parameters of the rf structure were $S_{11}=-26.79 \mathrm{~dB}$ and $S_{21}=-0.085 \mathrm{~dB}$, respectively. The total loss was $1.7 \%$, including $1.4 \%$ loss of two $\mathrm{TE}_{01}$ mode converters. The supporting rf structure is almost perfectly matched. When the bulk silicon, with oxide but without diode and metal structure on the surface, was placed, the $S$ parameters were $S_{11}=-2.500 \mathrm{~dB}$

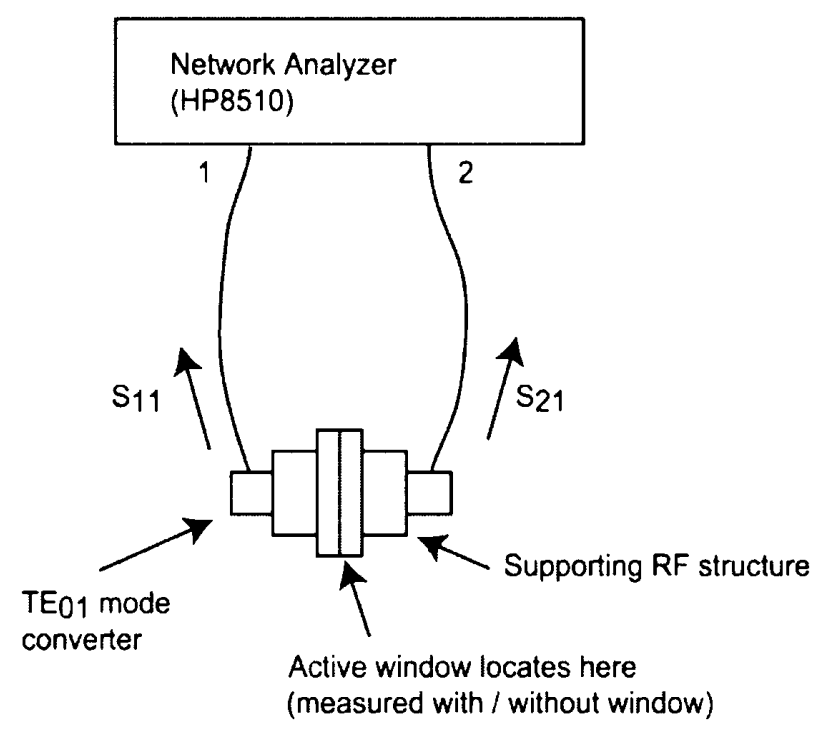

FIG. 13. Schematic diagram of the network analyzer measurement. 
and $S_{21}=-3.9872 \mathrm{~dB}$. The total loss was $3.9 \%$ including the loss of mode converters. The loss at the window was $2.5 \%$. This result is consistent with theoretical calculations. When the active window with full $p-i-n / n-i-p$ diode array structure on both sides of the window was inserted, the $S$ parameters were $S_{11}=-2.429 \mathrm{~dB}$ and $S_{21}=-4.1726 \mathrm{~dB}$. The total loss was $4.5 \%$, and the loss at the window was $3.1 \%$. These $S$ parameters are very close to those of the bulk silicon window. The difference between the losses of the window without and with the diode structure was $0.6 \%$. This $0.6 \%$ of power was dissipated into the heavily doped diode structure. Hence, we confirmed that the reflection caused by the radial line structure with the coverage factor of $10 \%$ is reasonably small.

The schematic diagram of the active switching experiment is shown in Fig. 14. The rf signals generated by the signal generator are amplified to $1 \mathrm{~W}$, and guided to the active window through an isolator and directional couplers. The reflection and the transmission of incident rf signals are picked up through $20 \mathrm{~dB}$ directional couplers and measured by the peak power meter (HP8990A). The voltage across the diode window is measured. Also, a current transformer measures the current through the diode window.

Prior to the active measurement, the characteristics of the rf components in the test setup were checked by the network analyzer. The measurement results are shown in Table II. The isolator had $-34.08 \mathrm{~dB}$ reflection coefficient at the port facing the active window. Thus, it was confirmed that there is no parasitic resonance in the test setup caused by the reflection of the components.

The $10^{16} \mathrm{~cm}^{-3}$ doping density version was used for this experiment. The typical waveforms with the various forward bias and zero reverse bias are shown in Figs. 15-17.

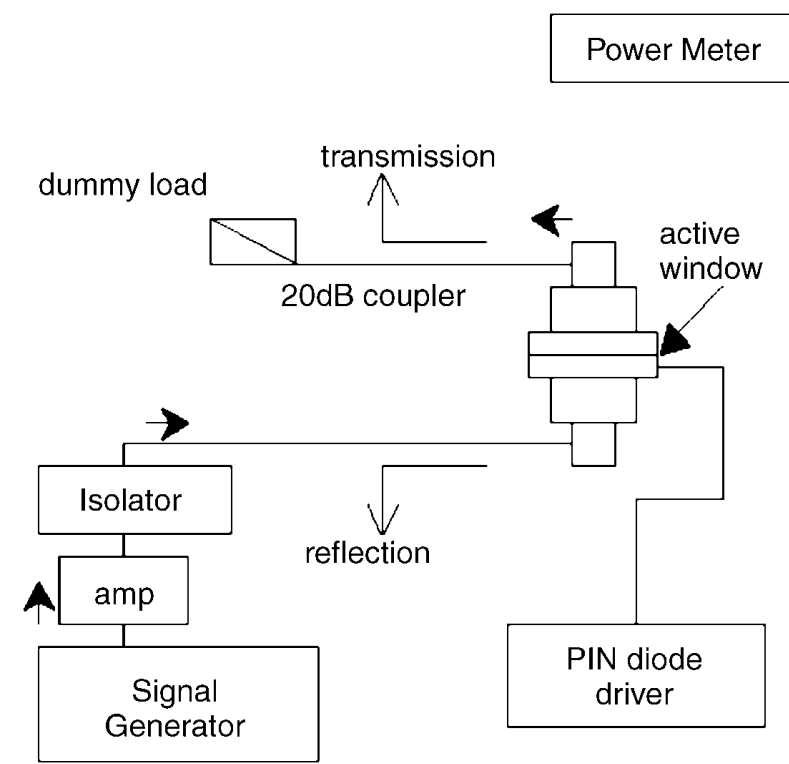

FIG. 14. Schematic diagram of the low power measurement.
TABLE II. Characteristics of the components.

\begin{tabular}{lc}
\hline \multicolumn{1}{c}{ Component } & Characteristics \\
\hline Isolator & Forward direction is port $1 \rightarrow$ port 2 \\
& $S_{11}=-33.45 \mathrm{~dB}$, \\
& $S_{21}=-0.74 \mathrm{~dB}$, \\
& $S_{12}=-32.12 \mathrm{~dB}$, \\
& $S_{22}=-34.08 \mathrm{~dB}$ \\
Terminator & $-63 \mathrm{~dB}$ reflection \\
Directional coupler 1 & Power pickup: $-19.9 \mathrm{~dB}$ \\
& reflection: less than $-40 \mathrm{~dB}$ \\
Directional coupler 2 & Power pickup: $-20.27 \mathrm{~dB}$ \\
& reflection: less than $-50 \mathrm{~dB}$ \\
\hline \hline
\end{tabular}

In these figures, the horizontal axis is time. The $S$ parameters of the total test set, including the active window, the rf structure, and the $\mathrm{TE}_{01}$ mode converters, are plotted.

As shown in the figures, when the forward bias voltage was applied, the carriers were injected into the intrinsic region and the conductivity of the intrinsic region increased significantly. As a result, the reflection from the active window increased and the transmission decreased. The rise time of the reflection and the transmission modulation is dominated by the speed of the diffusion process. It showed weak dependency on the applied voltage.

When the forward bias was turned off, the transmission and the reflection recovered to the initial state slowly. The natural recovery time of the transmission was about $50 \mu \mathrm{s}$.

The maximum forward bias current of $115 \mathrm{~A}$ was achieved. With this maximum forward current, the total reflection was modulated to $-0.6 \mathrm{~dB}$ and the total transmission was modulated to $-22 \mathrm{~dB}$. Comparing with the result of the network analyzer measurement described in the above, $S_{11}=-2.429 \mathrm{~dB}, S_{21}=-4.1726 \mathrm{~dB}$, the maximum transmission modulation was $18 \mathrm{~dB}$.

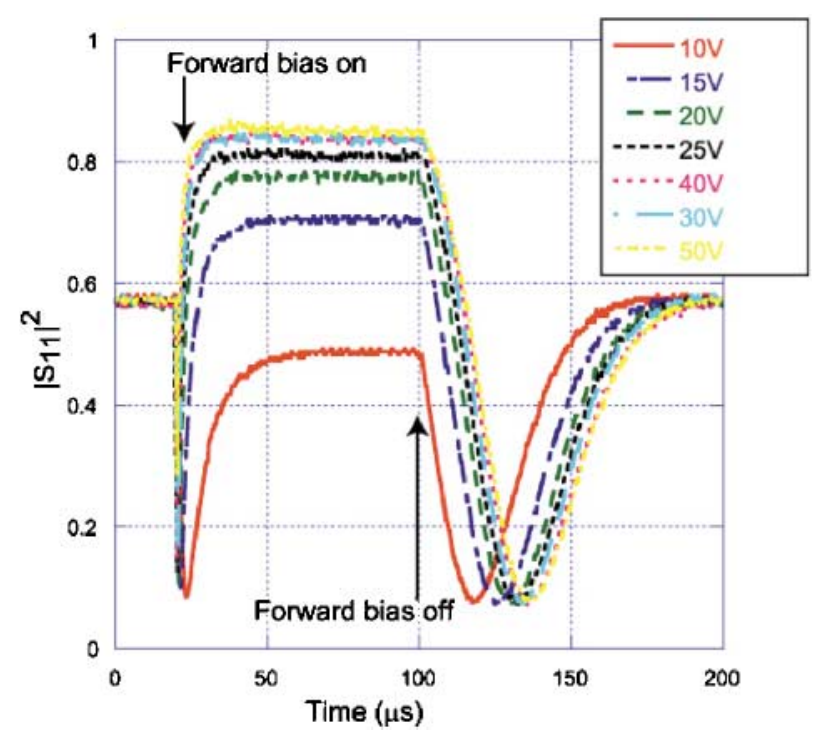

FIG. 15. (Color) Typical waveform of reflection with various forward biases. 


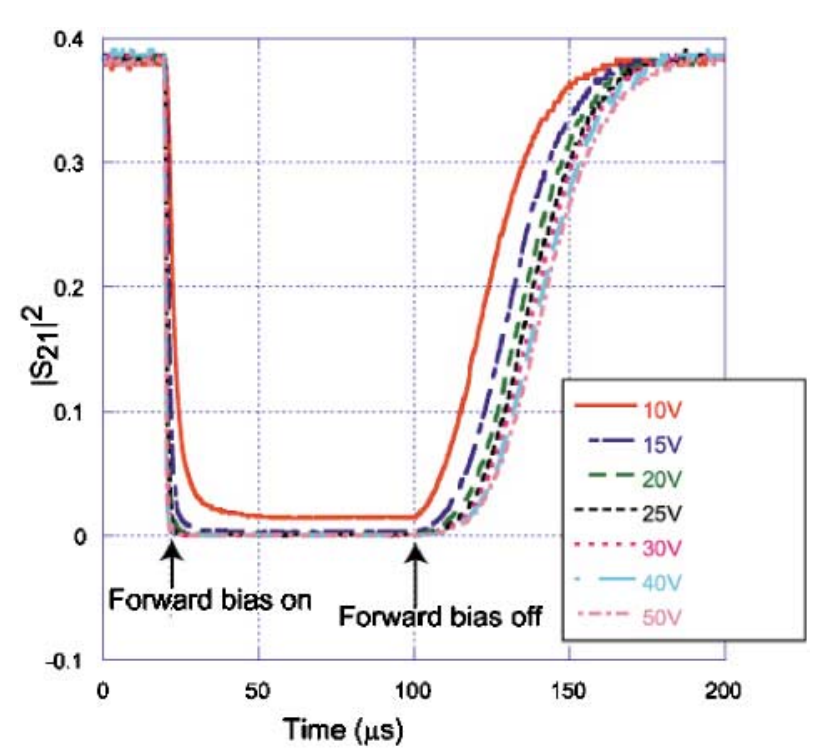

FIG. 16. (Color) Typical waveform of transmission with various forward biases.

The total loss with the $115 \mathrm{~A}$ forward bias current was $13.9 \%$ including the $1.4 \%$ loss of the $\mathrm{TE}_{01}$ mode converters. Hence, the loss dissipated into the active window was $\sim 11.5 \%$. From the measured loss of the active windows, the conductivity of the intrinsic region is calculated as $13.8 \Omega / \mathrm{cm}$ assuming a uniform distribution of the carriers in the intrinsic region. This conductivity corresponds to a carrier density of $7 \times 10^{14} \mathrm{~cm}^{-3}$.

In Fig. 18, the waveforms with a zero bias and a reverse bias at $-120 \mathrm{~V}$ are plotted. As shown in the figure, the switching time was improved from $50 \mu$ s with no reverse bias to $20 \mu$ s with the $-120 \mathrm{~V}$ reverse bias. When the transmission is close to zero, the curve of the transmission

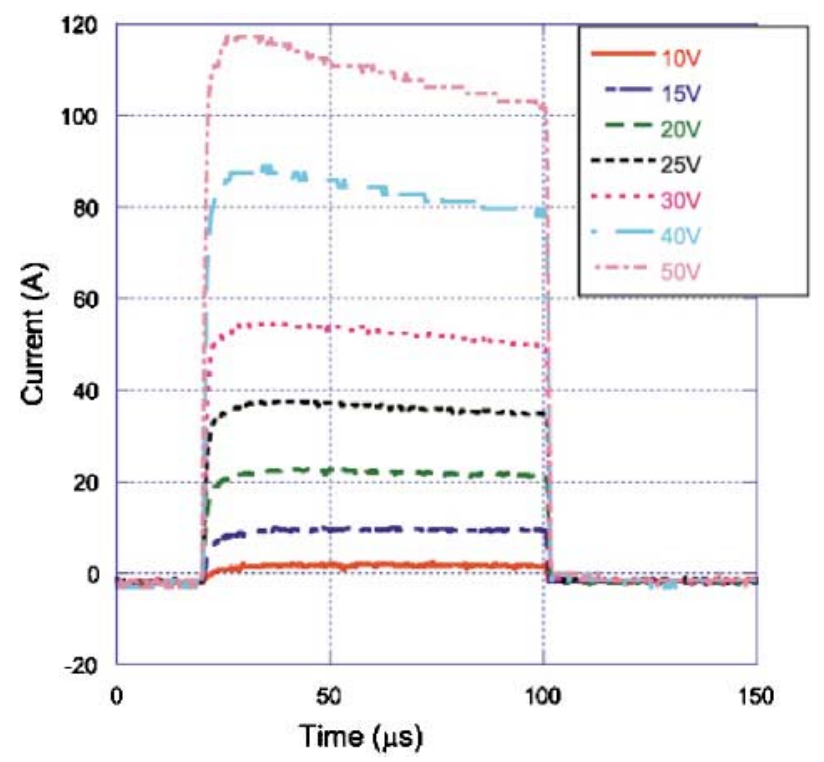

FIG. 17. (Color) Current with various forward bias voltages. recovery without and with the reverse bias is almost the same. This means that the effect of the reverse bias voltage is not much when the intrinsic region is filled with the injected carriers. The reverse bias is effective after the relative transmitted power exceeds 0.05 ; the reverse bias voltage sweeps the carriers.

This result can be explained as follows. When the intrinsic region is filled with a lot of carriers, the $p-i-n$ diode has very low resistance. Hence, most of the voltage drop occurs at the external resistance of the circuit, which includes (i) the resistance of the biasing circuit, (ii) the contact resistance between the metal springs and the surfaces of the active window and the rf structure, and (iii) the resistance of the thin metal lines on the surface of the window. The diode feels a low voltage and the electric field in the diode to sweep the carriers is small. In this situation, the carrier sweeping by the reverse bias voltage is not efficient. When the carrier density in the intrinsic region becomes relatively low, the voltage drop across the diode becomes higher. Thus, the carriers can be swept by the reverse bias faster than the case of zero bias. Therefore, reducing external resistance is necessary to sweep the carriers in the intrinsic region faster.

The $10^{17} \mathrm{~cm}^{-3}$ doping density version was also tested. However, the switching performance with the forward bias was not as good as the $10^{16} \mathrm{~cm}^{-3}$ version. At $60 \mathrm{~V}$ forward bias, the forward bias current was only $30 \mathrm{~A}$ and only
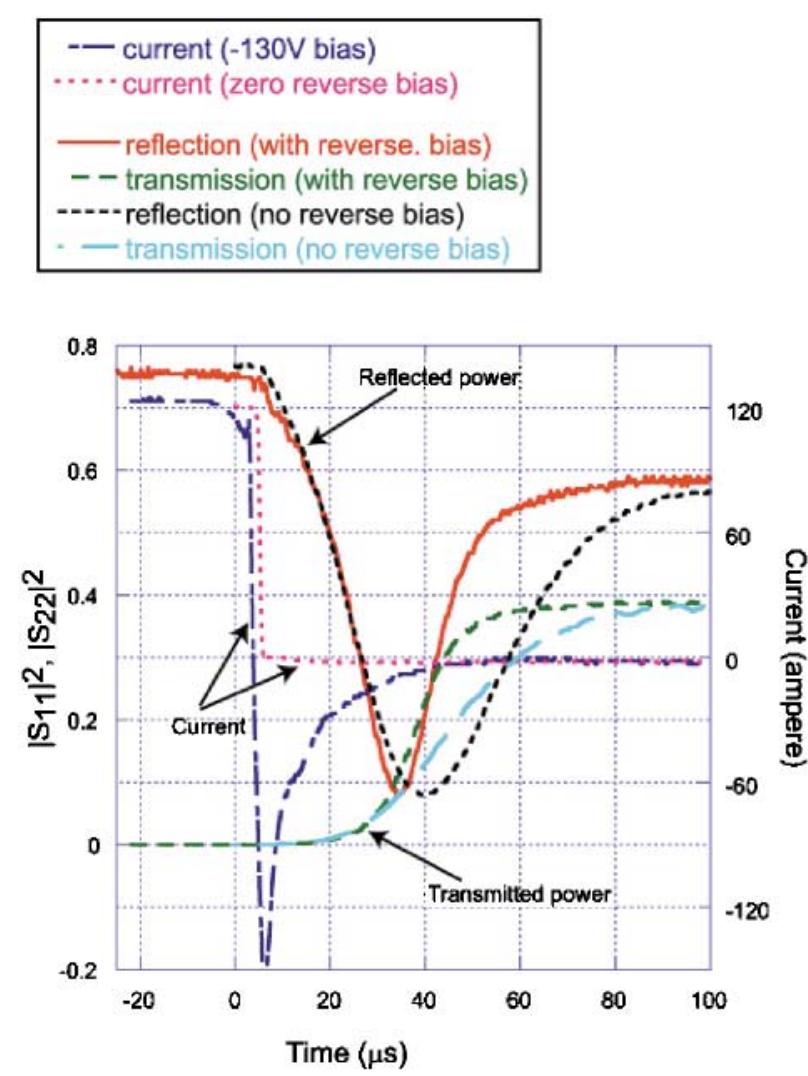

FIG. 18. (Color) The recovery from forward bias to reverse bias. 
$50 \%$ transmission modulation was achieved. It is quite possible that this was because of large contact resistance between the aluminum lines and the diode structure caused by processing problems. The highly likely scenario is that the natural oxide was not removed completely when aluminum was sputtered onto the silicon wafer.

As described above, the loss dissipated into the active window when the window is on was $11.5 \%$ and the achieved carrier density was $7 \times 10^{14} \mathrm{~cm}^{-3}$. The fastest switching speed from forward to reverse bias was $20 \mu \mathrm{s}$. To meet the requirements for the application of active pulse compression systems, the loss should be lower and the switching speed should be faster.

In this experimental version of active window, a low doping density was chosen to avoid the avalanche breakdowns with the dc reverse bias. The achieved carrier density in the intrinsic region, $7 \times 10^{14} \mathrm{~cm}^{-3}$, is a reasonable value for the $10^{16} \mathrm{~cm}^{-3}$ doping level. If the doping density is increased to $10^{19} \mathrm{~cm}^{-3}$, many more carriers will be injected into the intrinsic region and the loss dissipated into the active window will be lower. In that case, the reverse bias should be applied to the window in a short pulse, because high reverse dc bias voltage will cause avalanche breakdown. Some possibilities to improve the switching speed of the active window will be discussed in conclusion section.

\section{B. High power experiments}

\section{Results of passive high power experiment}

As described in Sec. II, the power handling capabilities of one single active window directly determines the number of active elements required to handle a certain amount of power. The high power experiment is necessary to examine the feasibility of the application of the high power switches to rf pulse compression systems.

The high power experiment was performed by using the high power $X$-band klystron (XL-2) and SLED-II rf pulse compression system at SLAC. The schematic diagram of the high power experiment is shown in Fig. 19. The high

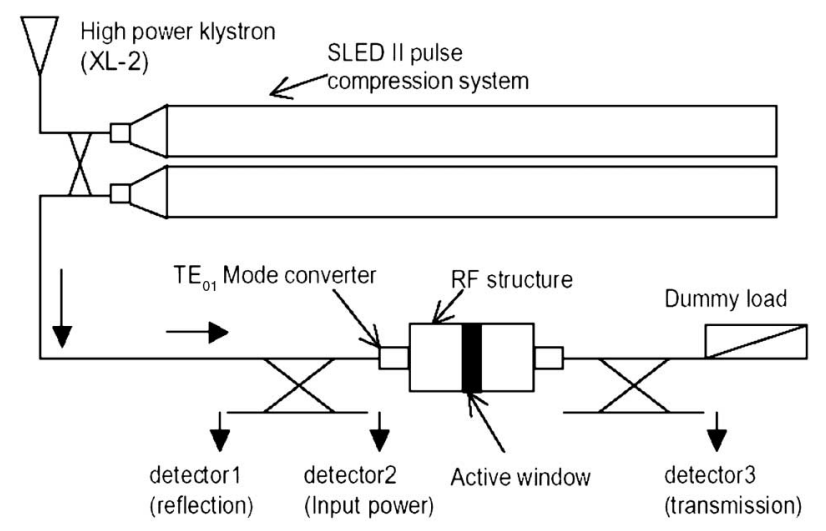

FIG. 19. Schematic diagram of the high power experiment. power rf signal of $900 \mathrm{~ns}$ pulse duration was generated by the klystron (the operating frequency is $11.424 \mathrm{GHz}$ ). The rf pulse was compressed by SLED-II pulse compression system. The compressed rf pulse was fed to the active waveguide window. Typical waveform of the SLED-II output with a dummy load is shown in Fig. 20. In the figure, the klystron output signal of $50 \mathrm{MW}$ is compressed to $200 \mathrm{MW}$. Since the window was not matched we used the pulse compression system to isolate the klystron from our window. Also it allowed us to operate the klystron at a relatively low power level so that it cannot be harmed by reflected rf power from the pulse compression system and the active window combination. The power level output from the pulse compression system to the active window was up to $15 \mathrm{MW}$. For our experiments, this power level was high enough. The pulse duration of compressed $\mathrm{rf}$ signal was $150 \mathrm{~ns}$. Since the active window did not have cooling systems, the repetition rate was limited to $5 \mathrm{~Hz}$.

The incident rf signal, the reflected signal, and the transmitted signal were measured by power meters and rf diode detector through directional couplers.

The $\mathrm{TE}_{01}$ mode converters have view ports to watch the surfaces of the window so that the video camera can detect flashing lights if arc occurs on the surface of the active window.

Two types of silicon windows were prepared for this high power experiment. The first silicon window is an active window, which has a full $p-i-n / n-i-p$ diode array structure on both the topside and the back side. This active window is the $10^{17} \mathrm{~cm}^{-3}$ doping density version. The resistivity of the base material is $5000 \Omega \mathrm{cm}$. The window thickness is $225 \mu \mathrm{m}$. The second silicon window had only the metal line structure on one side and no doping line structure; the other side had no structure (metal-only version). This version of the window was prepared for investigating the breakdown properties of the thin metal structure. The resistivity of the base material of this window is $1000 \Omega \mathrm{cm}$ and the thickness is $315 \mu \mathrm{m}$.

In Fig. 21, the reflected and transmitted powers from the active window with full diode structure are plotted. As shown in the figure, the reflected and transmitted powers were proportional to the input power. Hence, the reflection

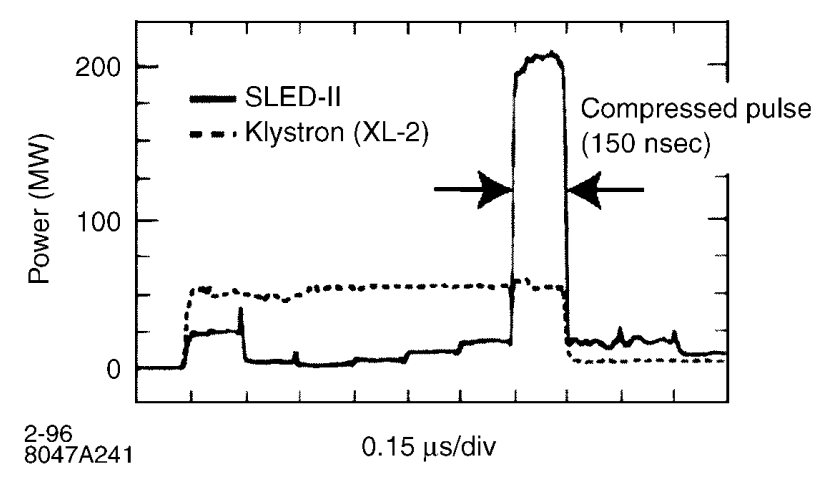

FIG. 20. SLED-II output signal. 

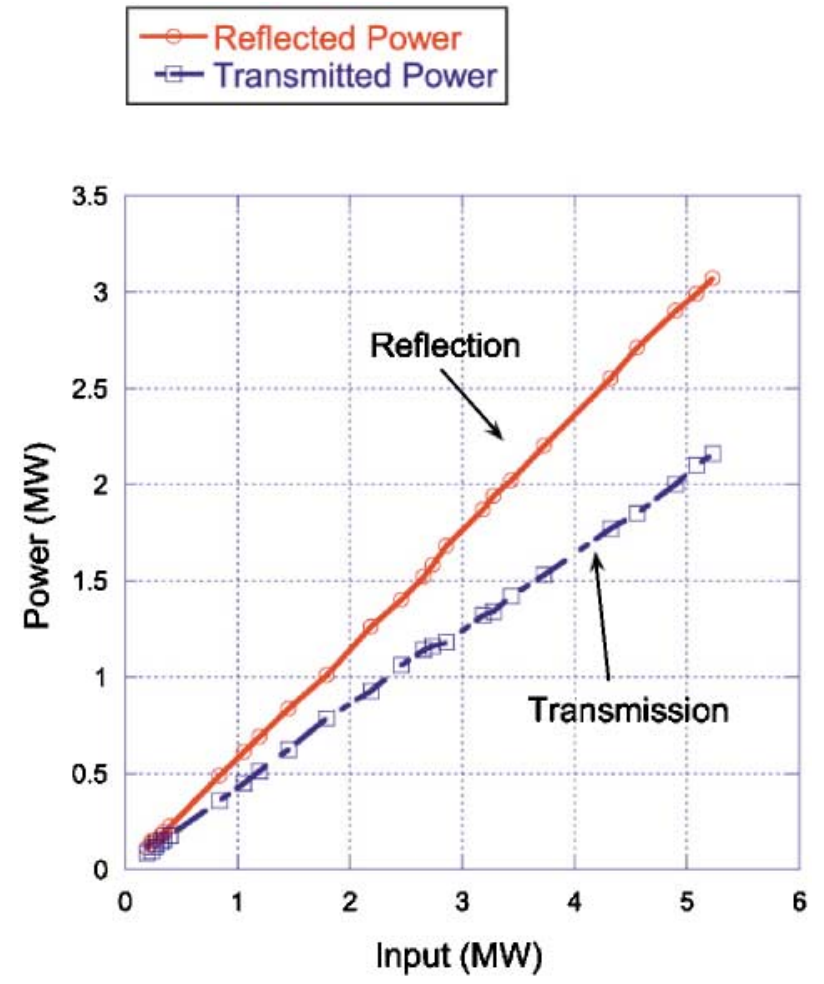

FIG. 21. (Color) Input versus transmission and reflection power.

coefficient did not change and the loss dissipated into the active window did not increase with increasing the input power up to $5.2 \mathrm{MW}$. This means that avalanche breakdowns did not occur at this power level. If the avalanche breakdown occurred, there would be a massive number of carriers in the intrinsic region, and reflection coefficient of the active window would have changed.

However, arcing started at the input power level of around $4 \mathrm{MW}$. When arcing occurred, the vacuum in the system became worse than about $10^{-5}$ Torr from $10^{-8}$ Torr at the normal high power operation, then the interlocks stopped the klystron. In Fig. 22, the waveforms of the transmitted and reflected rf signals when the arcing occurred are plotted. After the arcing started, the reflected power increased and the transmitted power decreased slowly.

This arcing was very vacuum dependent. We had to process slowly from the lower power levels after the trips. Flashing lights were observed by the video camera from both sides when the arcing occurred. These characteristics, vacuum dependency and flashing lights, are typical characteristics of the vacuum breakdowns, not the characteristics of avalanche breakdowns.

After achieving the maximum input power of $5.2 \mathrm{MW}$, the arcing occurred more and more frequently. Also, the arcing occurred at the lower power levels; i.e., we could not raise the input power any more. Thus, the high power operation was limited by this arcing. The maximum field at the surface of the window is calculated as $3.8 \mathrm{MV} / \mathrm{m}$ when the input power was 5.2 MW.

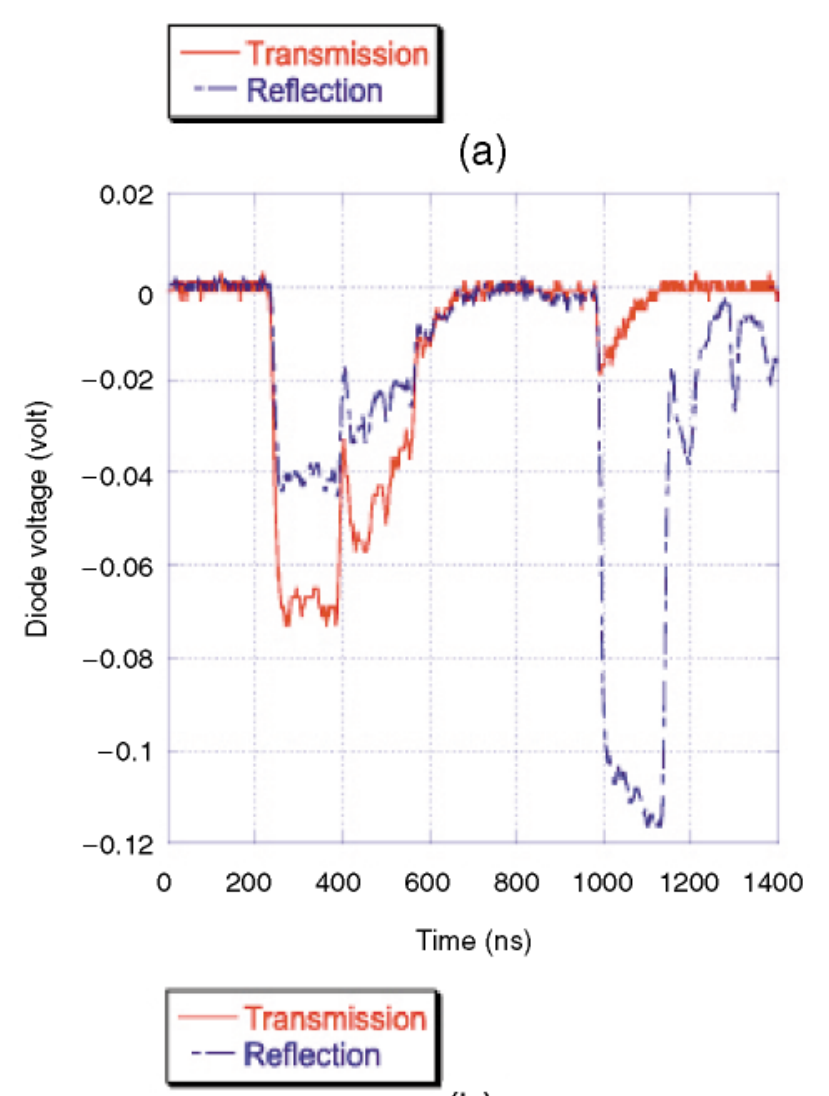

(b)

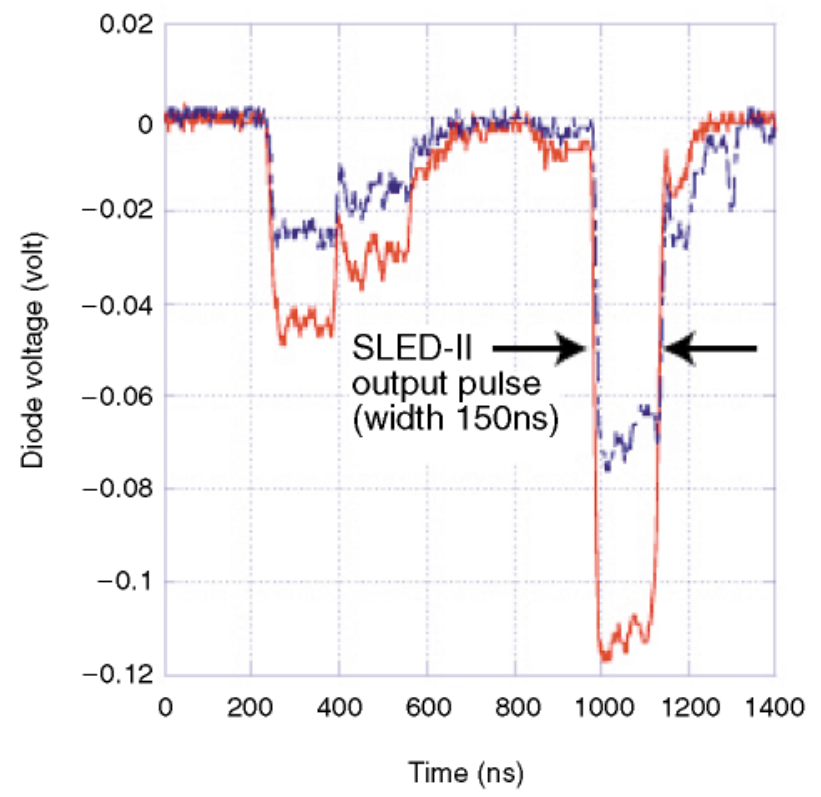

FIG. 22. (Color) Typical waveforms of reflected and transmitted rf signals when arc occurred. Bottom graph (b) shows waveform without arcing (metal-only version).

The pictures of the surface of the active window after this high power experiment are shown in Fig. 23. As shown in the figure, it is clear that the metal lines have many arcing traces but the silicon surface is not damaged at all. The arcing spots of the metal lines are similar to typical discharge traces. It is reasonable to assume that 


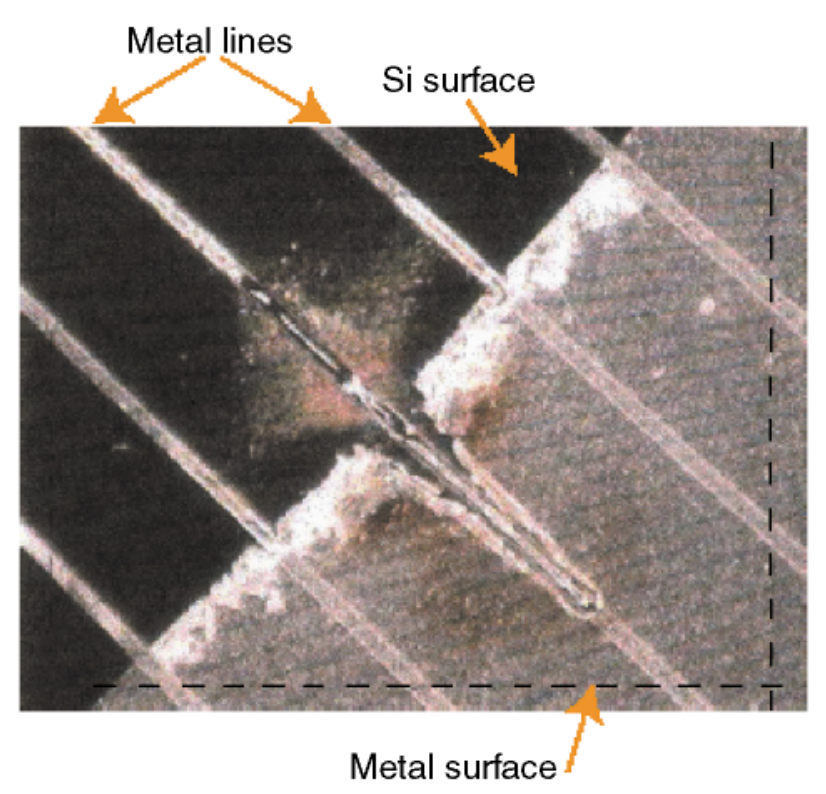

FIG. 23. Lines burned by biasing currents.

the arcing during the high power operation occurring between the metal lines and the vacuum became worse by aluminum vapor.

The metal-only version was also tested. The window was set in the system so that the downstream side had the metal structure. The metal-only version showed results similar to the active window with full $p-i-n / n-i-p$ diode structure. The arcing between the metal lines on the surface limited the high power operation. The maximum input power of $12 \mathrm{MW}$ was achieved. After the high power operation, similar arcing traces were observed on the metal lines. The silicon surface had no arc traces and no damage.

We tried the repetition rate of $30 \mathrm{~Hz}$ with the metal-only version. An interesting thing happened at the input power level of about $4 \mathrm{MW}$. The reflection increased significantly to almost full input power level, and it was kept high for long time (several minutes) even with much lower input power. We believe that, with $30 \mathrm{~Hz}$ of repetition rate, the window was heated to high temperature at several hundred degrees and the intrinsic carrier density in the silicon was increased by the thermal agitation. From this result, it is clear that, for the real application, some kind of cooling systems of the active window is necessary.

\section{Results of high power switching experiment}

As described above, the switching speed of the $p-i-n / n-i-p$ diode array active window is not fast enough to switch the SLED-II output pulse within the pulse duration. Hence, the forward bias current was started before the klystron pulse started, and was kept for the entire klystron pulse duration.

The waveforms of the transmitted and reflected rf signal with and without the forward bias voltage are shown in Fig. 24. With the forward bias voltage at $130 \mathrm{~V}$, the reflection increased and the transmission decreased. The reflected rf signal was modulated from 1.1 to $1.68 \mathrm{MW}$, and the transmitted rf signal was modulated from $0.63 \mathrm{MW}$ to $60 \mathrm{~kW}$. A $10 \mathrm{~dB}$ of transmission modulation was achieved. With the forward bias, no arcing on the surface of the active window was observed. It is reasonable because the active window is a conductor. When the window is biased in forward direction, the electric field of rf signal is minimum near the window surface.

However, after several hours of the active switching operation, the conductivity modulation became worse. Even with higher voltage, the forward current was not enough to modulate the reflection coefficient of the active window. Finally, no forward current would go through the active window.

The active window and the rf structure are connected to the dc biasing signal by metal springs. The contact is poor; the number of contact point touching the surface of the window and rf structure is the same as the number of curls in the spring, about a hundred. Also, each contact point has very small area. Hence, the total area of the contact is very small. The contacts became worse because the high forward current density destroyed them, then the forward bias could not go through.

Also, after the high power switching operation, several burned metal lines were found (see Fig. 23). The burned
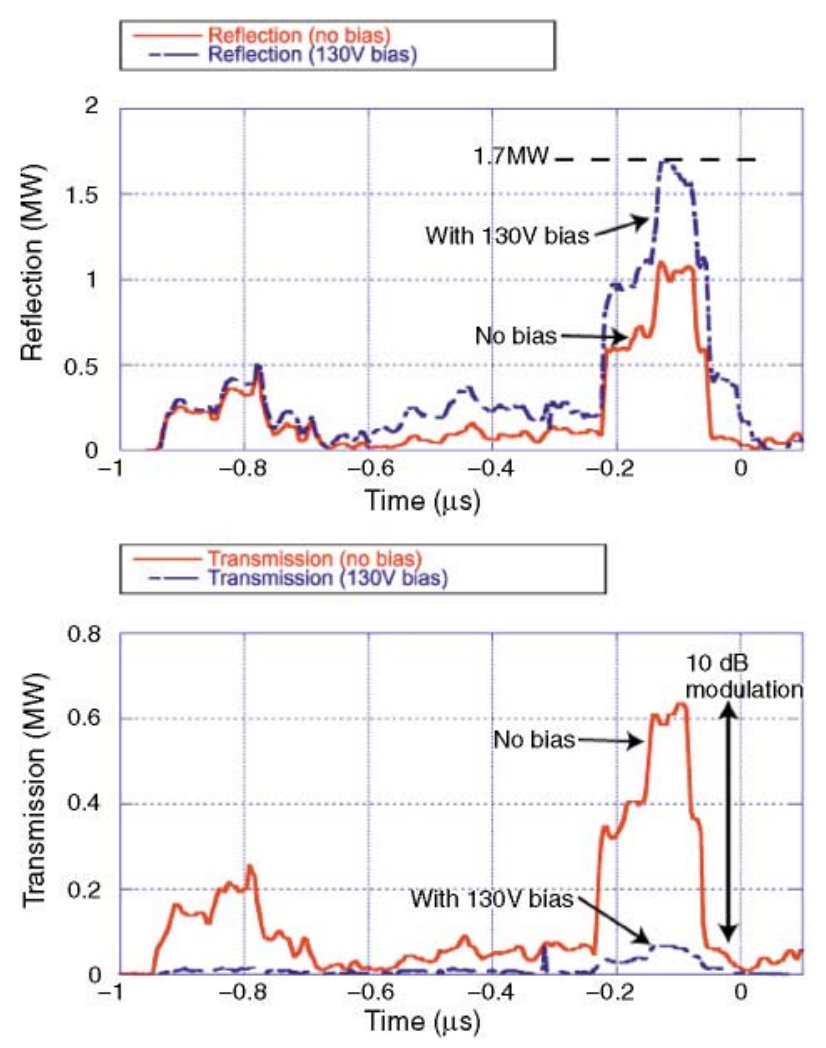

FIG. 24. (Color) Waveform with zero bias and $130 \mathrm{~V}$ forward bias. 
position is very close to the wall of the waveguide, where the electric field of $\mathrm{TE}_{01}$ mode is minimum. The biasing current burned them.

The high power switching operation was limited by the poor contact between the active window and the metal springs. Also, the thin aluminum lines were a problem for the forward biasing operation.

\section{CONCLUSIONS AND DISCUSSION}

In this paper, we have discussed high power microwave switches for the applications to the rf pulse compression systems of the future linear colliders. We described theoretical and experimental studies of high power microwave switches.

The design methodology of the high power SPDT switch, which consists of active elements, was developed. The active element is composed of a symmetrical tee junction, a short plane, and an active window. Two configurations of the SPDT switch were described: the SPST array and the cascaded phase shifter. In both configurations, the load of the rf power is distributed to several active elements. The maximum electric field at the active window when the window is off and the loss dissipated into the active window when the active window is on are important properties. Rigorous expressions for the maximum electric field and the loss were derived.

The scaling law, which governs the relation between the number of elements and the power handling capability, was developed. The number of elements is proportional to the loss at each window and inversely proportional to the square of the maximum electric field limit of the window. Hence, the maximum electric field of the active window dominated the necessary number of elements.

A new $p-i-n / n-i-p$ diode array active window were designed and built. The active window is built over a silicon substrate, which has the $p-i-n / n-i-p$ diode array structure on both surfaces of the substrate. The array structure on each side consists of 400 heavily doped lines covered by metal lines. The lines do not disturb the $\mathrm{TE}_{01}$ mode rf signals because all lines are perpendicular to the electric field lines of that mode. The processing of the active window is based on the standard and common IC processing. The rf structure was designed and built. The structure supports the active window at a designated position and gives the electrical contact for biasing. The low power measurement of the active window demonstrated $18 \mathrm{~dB}$ of transmission modulation. The losses dissipated into the active window were $11.5 \%$.

A high power experiment of the active window using a high power $X$-band klystron and a SLED-II pulse compression system was performed. Without bias, the active window was tested at up to $5.2 \mathrm{MW}$, to be compared with $100 \mathrm{~kW}$ of the power levels in past experiments. No avalanche breakdown in the silicon occurred. It has been confirmed that silicon active windows can handle rf sig- nals at the levels of multimegawatts. However, the arcing between the metal lines on the surface of active window limited the high power operation.

The switching speed could be improved by reducing the resistance of the circuit, by paralleling the biasing circuits, and by reducing the window thickness. Also, employing thick metal lines and covering the window surface with a dielectric layer can help reduce the arcing problem.

To conclude this study, we address the question: Is it possible to use silicon windows for switching very high power rf signals? The experimental results give the answer yes. Although more work is needed for real applications, it should be emphasized that silicon active windows can handle high power $X$-band rf signals at the power levels of multimegawatts.

\section{ACKNOWLEDGMENTS}

The authors thank Professor Ron Ruth, Professor P. Wilson, and Professor R. Miller for many useful dissections. We also thank Gordon Bowden for his help in constructing the rf structure. We are grateful for all the help we received from the SNF staff. We are also grateful for the help and effort of Chuck Yoneda and the Vacuum Group at the klystron Department at SLAC. This work was supported the U.S. Department of Energy Contract No. DEAC03-76SF00515.

[1] Sami G. Tantawi, in Proceedings of the International XX Linac Conference, Monterey, California, 2000, SLACR-561 (SLAC, Monterey, CA, 2000), pp. 673-677, and references therein.

[2] P. B. Wilson, Z. D. Farkas, and R. D. Ruth, in Proceedings of the Linear Accelerator Conference, Albuquerque, NM, 1990, SLAC-PUB-5330 (Los Alamos National Laboratory, Albuquerque, NM, 1990).

[3] Sami G. Tantawi, Ronald D. Ruth, and Arnold E. Vlieks, Nucl. Instrum. Methods Phys. Res., Sect. A 370, 297-302 (1993).

[4] S. Tantawi et al., Phys. Rev. ST Accel. Beams 5, 032001 (2002).

[5] S. Tantawi, in Proceedings of the Particle Accelerator Conference, Chicago, 2001 (IEEE, Piscataway, NJ, 2001)

[6] Sami G. Tantawi, Ronald D. Ruth, Arnold E. Vlieks, and Max Zolotorev, IEEE Trans. Microwave Theory Tech. 45(8), 1486-1492 (1997).

[7] S. M. Sze, Physics of Semiconductor Devices (Wiley, New York, 1981), 2nd ed.

[8] Joseph F. White, Microwave Semiconductor Engineering (Van Nostrand Reinhold, New York, 1982).

[9] Kenneth E. Mortenson, Jose M. Borrego, Paul E. Bakeman, Jr., and Ronald J. Gutmann, IEEE J. Solid-State Circuits 4(6), 413-421 (1969).

[10] Kenneth E. Mortenson, Albert L. Armstrong, Jose M. Borrego, and Joseph F. White, in Proceedings of the IEEE 59(8), 1191-1200 (1971).

[11] Sami G. Tantawi and Mikhail I. Petelin, IEEE MTT-S 
International Microwave Symposium Digest 2, 1153-1156 (1998).

[12] Fumihiko Tamura and Sami G. Tantawi, in Proceedings of the 7th European Particle Accelerator Conference, Vienna, 2000, http://accelconf.web.cern.ch/AccelConf/e00/index. html.

[13] Fumihiko Tamura and Sami G. Tantawi, in Proceedings of the International XX Linac Conference, Monterey, Califor- nia, 2000 (Ref. [1]).

[14] Robert E. Collin, Foundations for Microwave Engineering (McGraw-Hill, New York, 1992), 2nd ed.

[15] Stanford Nanofabrication Facility (SNF), http://snf. stanford.edu/.

[16] Sami G. Tantawi et al., IEEE Trans. Microwave Theory Tech 47(12), 2359-2546 (1999). 